\title{
Synchrotron high energy X-ray diffraction study of microstructure evolution of severely cold drawn NiTi wire during annealing
}

Cun Yu ${ }^{\mathrm{a}, \mathrm{d}}$, Bachir Aoun ${ }^{\mathrm{b}}$, Lishan Cui ${ }^{\mathrm{a}}$, Yinong Liu ${ }^{\mathrm{c}}$, Hong Yang ${ }^{\mathrm{c}}$, Xiaohua Jiang $^{\mathrm{a}}$, Song Cai ${ }^{\mathrm{e}}$, Daqiang Jiang ${ }^{\mathrm{a}, \mathrm{c}}$, Zunping Liu ${ }^{\mathrm{b}}$, Dennis E. Brown ${ }^{\mathrm{f}}$, and Yang Ren ${ }^{\mathrm{b}}$

${ }^{a}$ State Key Laboratory of Heavy Oil Processing and Department of Materials Science and engineering, China University of Petroleum, Beijing 102249, China

${ }^{b} X$-ray Science Division, Argonne National Laboratory, Argonne, IL 60439, USA

${ }^{c}$ School of Mechanical and Chemical Engineering, The University of Western Australia, Crawley, WA 6009, Australia.

${ }^{d}$ Shanghai Institute of Applied Physics, Chinese Academy of Sciences (CAS), Shanghai, 201800, China

${ }^{e}$ Fort Wayne Metals Research Products Corporation, 9609 Ardmore Avenue, Fort Wayne, IN 46809, USA

${ }^{f}$ Department of Physics, Northern Illinois University, De Kalb, Illinois 60115, USA

*to whom all correspondence should be addressed:

yinong.liu@uwa.edu.au

\begin{abstract}
Microstructure evolution of a cold-drawn NiTi shape memory alloy wire was investigated by means of in-situ synchrotron high-energy X-ray diffraction during continuous heating. The cold-drawn wire contained amorphous regions and nano-crystalline domains in its microstructure. Pair distribution function analysis revealed that the amorphous regions underwent structural relaxation via atomic rearrangement when heated above $100{ }^{\circ} \mathrm{C}$. The nano-crystalline domains were found to exhibit a strong cold work induced lattice strain anisotropy along $\langle 111\rangle$, which coincides with the crystallographic fiber orientation of the domains along the wire axial direction. The lattice strain anisotropy systematically decreased upon heating above $200{ }^{\circ} \mathrm{C}$, implying a structural recovery. Crystallization of the amorphous phase led to a broadening of the angular distribution of $<111\rangle$ preferential orientations of grains
\end{abstract}


along the axial direction as relative to the original $\langle 111\rangle$ axial fiber texture of the nanocrystalline domains produced by the severe cold wire drawing deformation.

Keywords: Shape memory alloy, Structural relaxation, Crystallization, in-situ synchrotron high energy $\mathrm{X}$ ray diffraction

\section{Introduction}

NiTi shape memory alloys (SMAs) are known for their functional properties such as the shape memory effect and pseudoelasticity. Both properties originate from martensitic transformations occurring in these alloys. Being a mechanical lattice distortion process as well as a phase transformation, the martensitic transformation is sensitive to the metallurgical and mechanical conditions of the alloy matrix, thus understanding these materials requires knowing the thermal and mechanical treatment history. NiTi alloys are often subjected to severe cold working during material production to control, or tune, their properties. It is known that NiTi has a high tendency to be amorphized upon cold working [1-3]. Therefore, a knowledge of the structural evolution of amorphized NiTi upon heating is of fundamental importance for NiTi materials design and processing. In addition, nano-crystalline NiTi SMAs have also attracted much attention in recent years because their transformation behavior and mechanical property characteristics are quite different than the conventional coarse-grained NiTi SMAs [4-10]. A feasible processing technique for large quantity production of nano-crystalline NiTi SMAs is to anneal amorphous NiTi formed after severe plastic deformation (SPD). Common techniques used for severe plastic deformation include cold rolling [3], high pressure torsion [11] and wire drawing [12].

Annealing upon heating of severely deformed NiTi alloys involves structural relaxation, crystallization and grain growth [13]. Some studies have been conducted to understand the kinetics of crystallization and grain growth, which has a significant influence on microstructure control, transformation behavior and the mechanical properties of NiTi alloys [13-15]. Peterlechner et al. studied the crystallization kinetics of cold-rolled amorphous NiTi [13]. They found that the nucleation and grain growth kinetics obey the Johnson-Mehl-Avrami (JMA) model, which is typical for a diffusion controlled process. Delville et al. studied the process of grain growth of cold drawn amorphous NiTi wires during pulsed electric current annealing by 
means of in-situ transmission electron microscopy (TEM) studies. They observed very fast grain growth within a few milliseconds (e.g., up to $1 \mu \mathrm{m}$ growth within 14-18 ms) and found superelastic properties in partially crystallized microstructures having grain sizes in the range of 25-50 nm [15]. Peterlechner et al. carried out in-situ TEM studies of the grain size evolution in amorphous NiTi produced by high pressure torsion [16]. They found that the NiTi alloy deformed by high pressure torsion contained medium-range-ordered (MRO) domains on the order of 1-3 nm dispersed in a continuous amorphous matrix, and that heterogeneous nanocrystallization occurred during heating via nucleation from these pre-existing MRO domains.

Whereas some knowledge has been accumulated of the crystallization and grain growth of amorphous NiTi upon heating, much less is known about the process of structural relaxation prior to crystallization. This is largely due to the technical difficulty in characterizing the structural features of an amorphous matrix. It has been reported that heating at low temperatures (e.g., $400{ }^{\circ} \mathrm{C}$ ) increases the Young's modulus and hardness of NiTi amorphous thin films, and both effects are attributed to the reduction of free volume in the matrix associated with relaxation caused by heating [17]. It has also been reported that the crystallization nucleation rate is increased after a pre-relaxation treatment, leading to a more homogeneous grain size distribution. This observation is attributed to the formation of shortrange ordered clusters during structural relaxation, which favor nucleation. However, it is not clear how the short-range ordered clusters are formed by atomic rearrangement [17]. Peterlechner et al. [13] studied the relaxation of cold rolled NiTi containing amorphous and highly deformed crystalline domains by means of modulated DSC measurements. They demonstrated that a commonly observed exothermic event upon continuous heating is associated with the relaxation of the amorphous phase. However DSC measurements cannot probe the physical structure and evolution of the relaxed amorphous state. In general, very little is known of the intrinsic behavior of relaxation or the structure of the relaxed alloy.

In this work, we studied the microstructure evolution, including structural relaxation, recovery, grain growth and texture evolution, of severely cold drawn NiTi wires during annealing by means of in-situ high energy X-ray diffraction and scattering. The experimental data were analyzed by means of the Williamson-Hall technique to elucidate the process of grain size evolution and internal stress relaxation of nanocryslline domains. Pair distribution function (PDF) measurements were used to characterize the structural relaxation and atomic shuffling of the amorphous phase during heating. 


\section{Experimental procedure and methods}

A $28 \mathrm{~kg}$ NiTi ingot with a nominal composition of $\mathrm{Ni}_{50.2} \mathrm{Ti}_{49.8}$ (at. \%) was prepared by vacuum induction melting. The ingot was hot forged at $850{ }^{\circ} \mathrm{C}$ into a $16 \mathrm{~mm}$ diameter rod and further hot drawn at $750{ }^{\circ} \mathrm{C}$ to a $2 \mathrm{~mm}$ diameter wire. The hot-drawn wire was then cold drawn to 0.5 $\mathrm{mm}$ in diameter at room temperature with intermediate anneals at $700{ }^{\circ} \mathrm{C}$. The $0.5 \mathrm{~mm}$ wire was subjected to a final cold drawing to $0.23 \mathrm{~mm}$ in diameter (corresponding to an area reduction of $79 \%)$.

Synchrotron high-energy X-ray diffraction (HE-XRD) was used to study the microstructure evolution of cold drawn NiTi wires during continuous in-situ heating. Experiments were performed on the 11-ID-C beam line of the Advanced Photon Source at Argonne National Laboratory (USA). High-energy $115 \mathrm{keV}$ X-rays $(0.10781 \AA$ ) of beam size $0.5 \mathrm{~mm} \times 0.5 \mathrm{~mm}$ were diffracted in transmission geometry towards a Perkin-Elmer large area detector to obtain two-dimensional (2D) diffraction patterns. The NiTi wires were placed $2 \mathrm{~m}$ from the PerkinElmer detector. 2D diffraction patterns were collected at $5{ }^{\circ} \mathrm{C}$ temperature intervals during heating. A Linkam-stage furnace heated the samples under an argon atmosphere at a rate of $5{ }^{\circ} \mathrm{C} / \mathrm{min}$. For each XRD measurement, heating was stopped to hold the sample at a constant temperature and the data were collected over a period of 30 seconds. The experimental set-up is shown schematically in Fig. 1.

The cold drawn NiTi wire specimen contained a large volume fraction of an amorphous phase, making it difficult to analyze its structure by means of conventional HE-XRD. To overcome this difficulty, we used pair distribution function (PDF) measurements to extract the atomic rearrangement before crystallization. In contrast to the conventional structural refinement based on Bragg intensities, PDF gives directly, in real space, the interatomic distances in a material by Fourier transforming the total scattering structure factor $S(Q)$. The commonly used reduced atomic PDF, $G(r)$, is defined as:

$$
G(r)=4 \pi r\left[\rho(r)-\rho_{0}\right]
$$

where $\rho(r)$ and $\rho_{0}$ are the local and average atomic number densities, respectively, and $r$ is the radial distance between two atoms. The $G(r)$ function exhibits peaks representing statistical 
characteristic distances separating pairs of atoms. Since the PDF contains both Bragg diffraction and diffuse scattering, the information about local arrangements is preserved but weighted by the scattering power of the pair of atoms.

PDF data analysis involves extracting the structure factor [18]:

$$
S(Q)=1+\left[I^{\text {coh. }}(Q)-\sum c_{i}\left|f_{i}(Q)\right|^{2}\right] /\left|\sum c_{i} f_{i}(Q)\right|^{2}
$$

where $I^{\text {coh. }}(Q)$ is the intensity of coherent scattering, $c_{i}$ is atomic concentration and $f_{i}(Q)$ is the $\mathrm{x}$-ray scattering factor of the $i^{\text {th }}$ atomic species. The atomic pair distribution function, $G(r)$, is obtained by taking a Fourier transform of the structure factor:

$$
G(r)=\frac{2}{\pi} \int_{Q=0}^{Q_{\max }} q[S(Q)-1] \sin (Q r) d Q
$$

where $Q$ is the magnitude of the wave vector $(Q=4 \pi \sin \theta / \lambda), 2 \theta$ is the angle between the incoming and outgoing $\mathrm{x}$-rays and $\lambda$ is the wavelength of the $\mathrm{x}$-ray.

The experimental set-up for PDF measurements is similar to that for in-situ high-energy X-ray diffraction, except that the detector is placed much closer to the specimen (400 mm away) in order to reach a larger reciprocal space.

The Williamson-Hall method [19] was used to determine grain size and lattice strains upon heating. Peak positions $(2 \theta)$ and peak full widths at half maximum $\left(\mathrm{FWHM}=\left(\beta_{\mathrm{hkl}}\right)_{\text {measured }}\right)$ were determined by Gaussian fitting the peak profiles. The instrumental contribution to line broadening was determined using a lanthanum hexaboride $\left(\mathrm{LaB}_{6}\right)$ standard. The instrumentcorrected broadening $\left(\beta_{\mathrm{hkl}}\right)$ was computed as:

$$
\left(\beta_{h k l}\right)=\left[\left(\beta_{h k l}\right)_{\text {measured }}^{2}-\beta_{\text {instrumental }}^{2}\right]^{0.5}
$$

In the Williamson-Hall method, it is assumed that the line broadening $\beta_{\mathrm{t}}$ of a Bragg reflection (h k l) originating from the small crystallite size follows the Scherrer equation $\beta_{t}=K \lambda /(\tau$ $\left.\cos \theta_{\mathrm{hkl}}\right)$. Here $\mathrm{K}$ is the shape factor, $\theta_{\mathrm{hkl}}$ is the Bragg angle and $\tau$ is the effective crystallite size normal to the reflecting planes. The strain-induced broadening $\beta_{\varepsilon}$ is given by the Wilson formula as $\beta_{\varepsilon}=4 \varepsilon \tan \theta_{\mathrm{hkl}}$. Here $\varepsilon$ is the root mean square value of the micro-strain. Assuming that the particle size and strain contributions to line broadening are independent of each other 
and both have a Cauchy-like profile, the observed line width (after correcting for instrumental broadening) is simply the sum of the two, i.e.,

$$
\beta_{h k l}=\beta_{t}+\beta_{\varepsilon}=\frac{K \lambda}{\tau \cos \theta_{h k l}}+4 \varepsilon \tan \theta_{h k l}
$$

or

$$
\beta_{h k l} \cos \theta_{h k l}=\left[\frac{K \lambda}{\tau}\right]+\left[4 \varepsilon \sin \theta_{h k l}\right]
$$

From a linear plot of of $\beta_{h k l} \cos \theta_{h k l}$ verses $\sin \theta_{h k l}$, the microstrain $\varepsilon$ and crystallite size $\tau$ can be estimated from the slope and the intercept of the line.

TEM samples were prepared by mechanically grinding and ion polishing the samples. Microstructure analysis was carried out using an FEI Tecnai F20 TEM operating at an accelerating voltage of $200 \mathrm{kV}$. Crystallization behavior of the wire was characterized using a Netzsch Phoenix 204 differential scanning calorimeter heating at a rate of $10{ }^{\circ} \mathrm{C} / \mathrm{min}$.

\section{Results}

\subsection{Microstructural and calorimetric features}

Fig. 2 shows TEM images of a NiTi wire before and after crystallization. Fig. 2a is a high resolution TEM image of the wire before annealing. The sample contained crystalline nanodomains (indicated by the yellow circles) embedded in a continuous amorphous phase. The volume fraction of the nanocrystalline domains surviving the cold working may be roughly estimated to be $<10 \%$ based on the TEM observations and synchrotron XRD measurements. The inset shows a selected area electron diffraction (SAED) pattern of the sample, demonstrating typical characteristics of an amorphous structure. The faint intensity heterogeneity along the diffused ring is from the remaining nanocrystalline domains. Fig. $2 b$ shows the microstructure of a specimen after annealing at $350{ }^{\circ} \mathrm{C}$ for $2 \mathrm{~min}$. The sample has partially crystalized. The grain size is mostly below $15 \mathrm{~nm}$. Amorphous regions are still present in large patches and also in between the nanograins. Fig. 2c shows the microstructure of a specimen after annealing at $450{ }^{\circ} \mathrm{C}$ for $2 \mathrm{~min}$. The amorphous domains in the cold worked matrix has fully crystallized. The grain sizes are mostly between $30 \sim 40 \mathrm{~nm}$. Fig. $2 \mathrm{~d}$ is a DSC curve of the cold drawn NiTi wire upon heating from the ambient temperature to $500{ }^{\circ} \mathrm{C}$. It is seen that a continuous exothermic event commences at $\sim 125^{\circ} \mathrm{C}$ until a larger exothermic peak starts at $335{ }^{\circ} \mathrm{C}$. The first event starting at $\sim 125{ }^{\circ} \mathrm{C}\left(\mathrm{T}_{\mathrm{r}}\right)$ is attributed to structural relaxation, 
and the strong exothermic peak starting at $335^{\circ} \mathrm{C}\left(\mathrm{T}_{\mathrm{x}}\right)$ is attributed to crystallization of the amorphous phase. The progressive relaxation (like a second order transition) and the final crystallization (as a first order transition) are two consecutive steps to convert the amorphous phase, which is in a metastable state, to the B2 crystalline state, which is thermodynamically more stable. Both steps are expected to release the internal energy of the amorphous phase, thus they give rise to a progressive and a latent heat exothermic event in the DSC measurement, respectively.

\subsection{Atomic rearrangement during structural relaxation}

Fig. 3 shows a high angle $\mathrm{x}$-ray scattering analysis of cold drawn $\mathrm{NiTi}$ at $25^{\circ} \mathrm{C}$. Fig. $3 \mathrm{a}$ is a close range (400 mm sample-detector distance) 2D diffraction pattern and a corresponding $360^{\circ}$ integrated $I(Q)$ intensity spectrum. Fig. $3 b$ shows the structure factor $S(Q)$ as a function of the scattering wave vector. The $\mathrm{S}(\mathrm{Q})$ function is calculated from $\mathrm{I}(\mathrm{Q})$ using equation (2). The $\mathrm{S}(\mathrm{Q})$ profile displays multiple peaks with the strongest first peak appearing at $\mathrm{Q}_{1}=2.973$ $\AA^{-1}$, followed by progressively decaying peaks towards higher scattering vector values. These are characteristic of an amorphous structure in which no long-range order is present. The inset shows the first structure factor maximum $\mathrm{Q}_{1}$ (solid line) and its Gaussian function fit (dotted line). It is also prudent to point out that the microstructure of the cold drawn wire is expected to be inhomogeneous across the cross-section, but this is beyond the detection of the synchrotron XRD technique used, which collects the average structural information across the entire section of the cold drawn wire.

Fig. 3c shows a plot of $\left(\frac{Q_{1}\left(T_{0}\right)}{Q_{1}(T)}\right)^{3}$ as a function of heating temperature. A change in $\mathrm{Q}_{1}$ implies a change in the average interatomic distance, which can be related to the change in the mean atomic volume [20] through

$$
\left(\frac{Q_{1}\left(T_{0}\right)}{Q_{1}(T)}\right)^{3}=\frac{V(T)}{V\left(T_{O}\right)}=1+\gamma\left(T-T_{0}\right)
$$

where $\mathrm{T}_{0}$ is a reference temperature (in this work, $25^{\circ} \mathrm{C}$ ), $\mathrm{V}$ is the mean atomic volume, and $\gamma$ is the volumetric coefficient of thermal expansion. Given that $\mathrm{Q}_{1}$ is a parameter in reciprocal space, $\left(\frac{Q_{1}\left(T_{0}\right)}{Q_{1}(T)}\right)^{3}$ is proportional to $\frac{V(T)}{V\left(T_{O}\right)}$. This relationship has been successfully applied to quantify free volume changes during the structural relaxation of bulk metallic glasses (BMG), 
their glass transition temperatures $\mathrm{Tg}$, and structural relaxations during annealing of oxide glasses [21].

It is evident in Fig. 3c that a slight change in the slope of the curve occurred at around $125^{\circ} \mathrm{C}$, which signifies a deviation from normal thermal expansion. This event coincides with the DSC observation of the exothermic event commencing at $125{ }^{\circ} \mathrm{C}$. This implies atomic rearrangements occurred upon heating, i.e., structural relaxation.

Fig. 4 shows a pair distribution function analysis of the structural evolution of the cold drawn NiTi wire upon heating. To extract the atomic rearrangement in real space during the structural relaxation, we calculated the reduced pair distribution function $G(r)$ via a sinusoidal Fourier transform of $S(Q)$ in the wave vector range of $0 \leq Q \leq 15 \AA^{-1}$ by applying equation (7). Fig. 4a shows the $\mathrm{G}(\mathrm{r})$ function of the cold drawn NiTi wire at $25^{\circ} \mathrm{C}$. $\mathrm{G}(\mathrm{r})$ provides information about how the atoms are distributed and displays peaks at positions where pairs of atoms are separated with high probabilities.

A reverse Monte Carlo simulation [22-24] based on the NiTi B2 structure is also shown in Fig. $4 \mathrm{a}$ (the red dashed curve) for comparison with the experimental result. The fitting was achieved by upsetting the NiTi B2 structure to an amorphous state (as shown in the inset Fig. 4a). It is seen that the local structure of the cold drawn NiTi is practically identical to the simulated amorphous structure. It is also apparent that the $G(r)$ peaks are asymmetric, implying overlapping of multiple peaks. Fig. $4 \mathrm{~b}$ shows an enlarged view of the first peak (R1) shown in Fig. 4a. This peak captures within its range two near neighborhood relationships in the NiTi B2 structure. The first one (at lower $r$ value) is for Ni-Ti (at $1 / 21 / 21 / 2$ coordinate) and the second one is for Ni-Ni or Ti-Ti (at 100 coordinate). A double Gaussian fitting was carried out to deconvolute the R1 peak into two peaks corresponding to these two nearest neighbor distances in the NiTi B2 structure. It is interesting to note that the position of r2' $(0.286 \mathrm{~nm})$ is smaller than the expected NiTi B2 value of r2 (0.296 nm—see Table. 1). This implies a closer packing in the local structure of the amorphous region as compared to the B2 crystalline state caused by the cold drawing.

The effect of heating on the nearest neighbor distances, as measured by the shifts of the G(r) peaks relative their original positions, is shown in Fig. 4c. The R1 peak shows a slight continuous increase with temperature, largely due to thermal expansion, and then levels off above $300{ }^{\circ} \mathrm{C}$. The bond lengths of R2 and R3 increased upon heating above $75^{\circ} \mathrm{C}$, suggesting 
short range atomic rearrangement. The bond lengths of R4 and R5 showed very little changes up to $200{ }^{\circ} \mathrm{C}$ and then decreased rapidly with further increasing temperature. One important aspect needing clarification is that each of these $G(r)$ peaks is a compound peak containing multiple interatomic pair distances which may be described using crystalline direction indices (refer to Table 1).

\subsection{Structural crystallization}

To study the crystallization behavior of the cold drawn NiTi wire upon heating, we performed in-situ high energy XRD measurements. These measurements help to reveal the crystallization process of the amorphous phase, the relaxation of residual strains in the nano-crystalline domains, and grain growth. Fig. 5 shows in-situ XRD measurements revealing the crystallization of the amorphous phase upon heating. Fig. 5a shows a few examples of the 2D diffraction patterns of the NiTi specimen recorded at different temperatures upon heating. The inner broad ring is caused by diffuse scattering from the furnace's quartz window. Fig. $5 \mathrm{~b}$ is a contour plot of 1D diffraction patterns (intensity vs. 2-theta) as a function of annealing temperature. Before heating, there exists diffuse scattering corresponding to the amorphous phase of NiTi (such as at (110)) as well as broad Bragg peaks at (110), (200) and (211) of the NiTi B2 structure. These are from the nano-crystalline domains of NiTi surviving the cold wire drawing. Upon heating above $330{ }^{\circ} \mathrm{C}$, more peaks from the B2 structure emerge, implying crystallization of the amorphous phase in the NiTi wire. Whereas this interpretation is purely based on the observed evidence, it should be pointed out that there could be a martensite phase among the nanocrystalline domains seen in Fig. 2a, as reported by Malard et al. [12]. However, this is beyond the detectability of the synchrotron XRD method used in this study.

\subsection{Residual strain relaxation}

Fig. 6 shows an XRD analysis of the evolution and anisotropy of the internal lattice strains in the nano-crystalline domains. Fig. 6 a shows the $2 \mathrm{D}$ diffraction pattern of the original state of the wire sample and Fig. 6e shows its corresponding azimuthal plot. It is seen that the B2 (110) reflection exhibits a wavy line along the azimuth angle (with $90^{\circ}$ corresponding to the axial direction of the wire), implying a spatial anisotropy of the B2(110) d-spacing, apparently due to internal lattice strains in the nano-crystalline domains. Upon heating to higher temperatures, the wavy line of the B2 (110) diffraction peak gradually straightened up (Fig. 6f-h), indicating a homogenization of the anisotropic internal strains. 
To characterize the internal strain anisotropy, the B2(110) d-spacing value was evaluated along the azimuthal direction at $12^{\circ}$ intervals. The results are shown in Fig. 7. The variation is fitted with a sine function having a period of $180^{\circ}$. The range of variation is $0.03 \AA$, corresponding to a lattice anisotropy strain of $1.3 \%$. Fig. $7 \mathrm{~b}$ shows B2(110) d-spacing profiles of samples heated to different temperatures. It is seen that the magnitude of the B2(110) d-spacing strain anisotropy decreased progressively with increasing temperature. The lattice strain anisotropy, defined as the d-spacing variation magnitude $\Delta \mathrm{d}$ relative to the mean $\mathrm{d}$-spacing $\mathrm{d}_{\mathrm{o}}$, is shown in Fig. 7c as a function of temperature. It is seen that the B2(110) lattice strain anisotropy decreased with increasing annealing temperature, with an increasing rate. This temperature is well below the onset temperature for crystallization but consistent with that for structural relaxation (Fig. 5b) of the amorphous phase. This implies that the cause of this reduction of internal strain anisotropy is due to a short range atomic rearrangement within the nanocrystalline domains.

\subsection{Nano grain growth and microstrain relaxation}

Grain size and lattice strains of the samples were determined from the XRD data (e.g., Fig. 5b) by means of the Williamson-Hall method. Fig. 8 shows the evolution of grain size and lattice strains with temperature after crystallization. Fig. 8a shows the relationship between $\beta \cos \theta$ and $4 \sin \theta$ for different temperatures, as per Eq. 6. The intercept at $4 \sin \theta=0$ and the slope of each line were obtained by a linear fit of the data from 6 diffraction peaks. The $2 \theta$ values for the peaks were obtained from integrating the $2 \mathrm{D}$ diffraction spectra over $360^{\circ}$ in the azimuthal direction and performing a Gaussian fitting of the resulting peaks. The average grain size and lattice strain of the nano-crystalline NiTi specimens are plotted in Fig. $8 \mathrm{~b}$ as a function of temperature. The average grain size was $\sim 15 \mathrm{~nm}$ after crystallization (at $\sim 330{ }^{\circ} \mathrm{C}$ ), and increased continuously with increasing heating temperature to $\sim 40 \mathrm{~nm}$ after heating to $450{ }^{\circ} \mathrm{C}$, consistent with the TEM results (Fig. 2c). In comparison, the crystalline matrix still contained an average lattice strain of $0.18 \%$ after crystallization. The lattice strain decreased continuously with heating to nearly nil $(<0.08 \%)$ after heating to $450{ }^{\circ} \mathrm{C}$.

\subsection{Texture evolution}

During crystallization and internal lattice strain relaxation, grain orientation is also found to change as a result of annealing. Fig. 9 shows inverse pole figures of the wire sample in the 
axial direction obtained at different temperatures upon heating. In total 72 different $x$-ray diffraction spectra were collected from the 2-D diffraction patterns, one for every $5^{\circ}$ in the azimuthal circle and then analyzed with the Rietveld method [25] using a program package called Materials Analysis Using Diffraction (MAUD) [26]. The procedure is similar to that described by Wenk et al. [27], using the EWIMV texture model. Data are refined by taking into account instrument parameters and background, and crystallographic and microstructural parameters. One aspect needing clarification is that, due to the shape memory nature of NiTi and the very thin wire diameter $(0.23 \mathrm{~nm})$ of the sample, there is a possibility of wire bending during in-situ heating. As a result, the wire axial direction relative to the $\mathrm{X}$-ray beam line may not be accurate to within a few degrees. This needs to be taken into account when analyzing and interpreting the orientation texture information.

Fig. 9(a) shows the texture of the nano-crystalline domains of the cold drawn sample. A strong fiber texture can be recognized in the drawing direction. The highest texture intensity appears between $\langle 223\rangle$ and $\langle 445\rangle$, within a narrow range of $\sim 5.2^{\circ}$. This texture remained unchanged with heating until crystallization of the amorphous regions at $330{ }^{\circ} \mathrm{C}$, as expected. After crystallization, a broader texture emphasis emerged between $\langle 223\rangle$ and $\langle 323\rangle$ and gradually intensified with increasing temperature.

\section{Discussion}

It is evident in Fig. $2 \mathrm{~d}$ that there is a continuous exothermic event starting at $\mathrm{T}_{\mathrm{r}}=125^{\circ} \mathrm{C}$ upon heating prior to crystallization at $\mathrm{T}_{\mathrm{x}}=335{ }^{\circ} \mathrm{C}$. A similar exothermic effect has also been observed for severely cold rolled NiTi, and the effect was attributed to a structural relaxation of the amorphous phase in the matrix [13]. In this study, we discovered that, within this temperature range, active atomic rearrangement occurred. These atomic rearrangement activities caused a volume expansion of the amorphous structure (Fig. 3c), structural relaxation of the amorphous regions (Fig. 4c) and lattice strain release in the nano-crystalline domains (Fig. 7b).

\subsection{Volume expansion}

The amorphous structure of the severely deformed NiTi matrix may be described as "random close packed". This is based on the concept that, when free to take positions within the structure, the atoms will tend to pack up in the closest form possible due to interatomic attraction, i.e., 
metallic bonding. Considering that the crystalline structure of NiTi is B2, which has an atomic packing density of 0.68 (neglecting atomic size differences between $\mathrm{Ni}$ and $\mathrm{Ti}$ ) that is less than the close packed structure (atomic packing density of 0.74 ), it is therefore expected that the amorphization of the B2 structure is accompanied with a volume contraction. This is consistent with the volume expansion ( $0.88 \%$, as seen in Fig. 3c) observed during structural relaxation upon heating to in the temperature range of $125-320^{\circ} \mathrm{C}$. Crystallization of the amorphous phase resulted in a further volume expansion of $0.97 \%$ (calculated from Fig. 3c).

\subsection{Atomic movement, structural relaxation and short range ordering}

The evidence shown in Fig. 4c demonstrates clearly that there is atomic movement within the amorphous matrix upon heating prior to crystallization. To reveal the mechanism of atomic movement during structural relaxation, we simulated the amorphization process of crystalline NiTi-B2 structure with a closed packed structure (FCC or HCP). The local atomic structure of bulk metallic glasses produced by rapid cooling is described as being "random close packed" $[28,29]$. The $\mathrm{G}(\mathrm{r})$ of amorphous $\mathrm{Ni}_{40} \mathrm{Ti}_{60}$ produced by severe plastic deformation is found to be almost identical to that of rapidly quenched amorphous $\mathrm{Ni}_{40} \mathrm{Ti}_{60}$ [30]. The atomic stacking sequence of $\mathrm{BCC}$ is $\mathrm{ABAB}$ in the $\langle 110\rangle$ direction, the close packed direction of the structure. This is similar to the stacking sequence of HCP in the [0001] direction, also the close packed direction of the structure. Both $\{110\}$ of BCC and $\{0001\}$ of HCP are closed packed planes. Therefore, it is highly plausible that the BCC structure will amorphize to an "HCP-like" randomly close packed structure. Considering the above, we simulated the amorphization of crystalline NiTi-B2 structure with an HCP-like structure by structural upsetting during cold drawing and atomic rearrangement upon heating (resulting in a subsequent structural relaxation).

Table 1 compares the atomic arrangements of ideal BCC and HCP structures within a short range of $\mathrm{r}<0.661 \mathrm{~nm}$ (from a random reference lattice point). A nominal atomic radius $(\mathrm{R})$ of $0.128 \mathrm{~nm}$ was used, which was chosen to correspond to the position of peak R1 ( $2 \mathrm{R}=0.256$ $\mathrm{nm}$ ) in Fig. 4a. The $\mathrm{r}$ value in the table represents the distance of an atom to the reference lattice point. For the HCP structure, the first row (No. of atoms) indicates the number of atoms for each of the Miller indices recorded in the third row (Direction indices). The second row (Total No. atoms) is the accumulated total number of atoms included within the sphere defined by the indices. For example, at $\langle 22 \overline{4} 0\rangle$, the number of atoms for this direction indices is 6 , the distance of these atoms to the reference atom at the origin is $r=0.512 \mathrm{~nm}$, and the total number 
of atoms within this radius (r) is 56. Similarly, the same information is also given for the BCC structure. The bottom row of the table lists the $\mathrm{R}$ values as determined in Fig. $4 \mathrm{a}$, placed here at the most proper positions relative to $r$.

With such information, atomic movements within the BCC structure when it converts to the HCP structure, as a proxy to its amorphization process, can be discussed. As shown in the table, the nearest neighboring atoms around the reference atom, i.e., the coordination number of a structure, is 12 in the HCP lattice (the $11 \overline{2} 0$ shell) and 8 in the BCC lattice (the 111 shell). This implies that, in the process of amorphization, some of the second nearest neighboring atoms in the BCC lattice (four of the six atoms in the 100 shell) will move inward to fill in the "vacant sites" to form a closer packed structure, as indicated by the red arrow in the table. The two remaining atoms in the 100 shell will move outward, as indicated by the blue arrow, to join the second shell of the close packed structure (the $\overline{4} 403$ shell in the HCP structure). By the same principle, all the required atomic movements in the BCC structure during the amorphization process can be determined, with some moving inward (marked by red arrows) and some moving outward (marked by blue arrows). Given the above, it is natural to understand that the reverse atomic movements will occur during structural relaxation and crystallization upon heating.

As seen in Table 1, the measured R2 G(r) peak corresponds to a position in between $\mathrm{r} 4$ (110) and r6 (311) shells in the BCC structure, and is contributed to by six atoms moving outward from the $\mathrm{r} 4$ shell and 12 atoms moving inward from the $\mathrm{r} 6$ shell. This implies that during relaxation upon heating, some atoms (12) will be moving outward and some (6) moving inward, as observed in Fig. 4b. The R2 value increased at lower temperatures and decreased at higher temperatures, reflecting the mixed atomic movements during relaxation. The R3 peak corresponds to $\mathrm{r} 6$ of the BCC crystal. This peak involves exclusively inward movement of $50 \%$ of the population of atoms in this shell during amorphization. Therefore, upon heating, the R3 value increased monotonically during structural relaxation, as evident in Fig. 4b. The R4 peak corresponds to 13 (the 210 shell). This peak is formed by expelling $50 \%$ of its population of atoms, including four inwards and eight outwards from the shell. During structural relaxation, the R4 position is expected to decrease accompanying the return of its lost atoms, as evident in Fig. 4c. 


\subsection{Internal stress relief}

An anisotropic residual strain was observed in the nano-crystalline domains embedded in the amorphous matrix, as evident in Fig. 7a. This internal strain appears high (possibly tensile) in the axial direction and low (possibly compressive) in the radial direction, apparently created by the severely cold wire drawing, which is an elongation in the axial direction and contraction in the transverse direction. Upon heating to above $200{ }^{\circ} \mathrm{C}$, the internal stress was relieved in both directions, leading to a progressive reduction of the residual strain anisotropy, as seen in Fig. 7b. It is also evident in Fig. 7c that the internal stress is relieved to nearly nil upon heating to the crystallization temperature, implying that the internal stress is created between the amorphous matrix and the nanocrystalline domains. The stress relief occurred within the temperature range where atomic rearrangement and structural relaxation occurred. In this regard, it is obvious that the stress relief is a result of atomic rearrangement activities expressed in Fig. 4c.

\subsection{Texture variation}

As shown in Fig. 9a, the cold drawn NiTi wire exhibited strong fiber texture near $\langle 223\rangle-\langle 445\rangle$ for its nano-crystalline domains. This texture orientation is $\sim 11^{\circ}-\sim 6^{\circ}$ away from the $\langle 111\rangle$ direction, which is more commonly observed for axial texture orientation of cold drawn NiTi wires [31-33]. This small discrepancy is believed to be related to the low sample geometry stability during heating owing to its shape memory nature and very thin sample diameter, as explained above. The actual axial texture orientation is believed to be consistent with the more common observation of $\langle 111\rangle$.

After crystallization, the original axial texture orientation was broadened, forming a new emphasis within $\langle 223\rangle \sim\langle 323\rangle$, a conical spread of about $10^{\circ}$. This highly oriented crystallization of the amorphous regions around the initial texture orientation implies strong influence of the pre-existing nano-crystalline domains.

As shown in Fig. 1a, the average size of nano-crystalline domains is $\sim 4 \mathrm{~nm}$ and the distance among them is $\sim 10 \mathrm{~nm}$. It was also measured that the average grain size after crystallization is $\sim 15 \mathrm{~nm}$ (Fig. 8b). Thus it is feasible that the crystallization process is actually a growth process of the pre-existing nano-crystalline domains. 


\section{Conclusions}

This study analyzed the microstructural evolution process of a cold drawn NiTi wire upon heating by means of in-situ high-energy X-ray diffraction and high resolution transmission electron microscopy. The main findings are summarized as follows:

1. The severely cold drawn NiTi wire contains largely an amorphous matrix with a small quantity of nanocrystalline domains surviving the cold work. The nanocrystalline domains have an apparent fiber texture within a narrow range between $<223>$ and $<445>$ along the axial direction of the wire. The amorphization of the NiTi-B2 structure is analogous to a densification process forming a random close packed structure.

2. The amorphous matrix experiences structural relaxation upon heating above $100{ }^{\circ} \mathrm{C}$. The structural relaxation is associated with a small volume expansion, a mild exothermic effect, and active atomic rearrangements. The atomic rearrangements occur in a reverse fashion to the amorphization densification process.

3. The amorphous matrix crystallizes at $335{ }^{\circ} \mathrm{C}$ upon heating. The newly formed crystals have a fiber texture around $\langle 223\rangle \sim\langle 323\rangle$, which is within a narrow $10^{\circ}$ conical angle range from the original textural orientation of the residual nanocrystalline domains surviving the cold work. This implies a guiding influence of the residual nanocrystalline domains in the crystallization texture of the amorphous matrix.

4. The residual nanocrystalline domains surviving the cold wire drawing exhibit an internal stress anisotropy, with tensile strains along the wire axial direction and compressive strains in the transverse direction. The internal strains are found to be relaxed upon heating, leading to a progressive reduction of the strain anisotropy. The internal stress relaxation occurs within the temperature range up to the crystallization temperature of the amorphous matrix. This demonstrates that the internal stress relaxation is a result of the atomic rearrangements of the amorphous matrix.

\section{Acknowledgments}

The authors wish to thank Prof. R. Wenk, Prof. L. Lutterotti and Dr. W Kanitpanyacharoen for their help with texture analysis. This work was supported by the National Natural Science 
Foundation of China (51231008, 11474362 and 51401240), the National 973 program of China (2012CB619403), the Australian Research Council (Grant No. DP140103805), and the Institute for NanoScience, Engineering, and Technology (INSET) of Northern Illinois University. Use of the Advanced Photon Source at Argonne National Laboratory was supported by the U.S. Department of Energy under contract No. DE-AC02-06CH11357.

\section{References}

[1]. Tat'yanin YEV, Kurdyumov VG, Federov VB, Obtaining an amorphous TiNi alloy during deformation by shear under pressure, Phys. Met. Metallogr. 62 (1986) 118-124.

[2]. Koike J, Parkin DM, Nastasi M, Crystal-to-amorphous transformation of NiTi induced by cold rolling, J. of Mater Res, 5 (1990) 1414-1418.

[3]. Peterlechner M, Waitz T, Karnthaler H P, Nanoscale amorphization of severely deformed NiTi shape memory alloys, Scripta Materialia, 60 (2009) 1137-1140.

[4]. Waitz T, Kazykhanov V, Karnthaler HP, Martensitic phase transformations in nanocrystalline NiTi studied by TEM, Acta Materialia, 52 (2004) 137-147.

[5]. Tsuchiya K, Hada, Y Koyano T, Nakajima K, Ohnuma M, Koike T, Todaka Y, Umemoto M, Production of TiNi amorphous/nanocrystalline wires with high strength and elastic modulus by severe cold drawing, Scripta Mater. 60 (2009) 749-752.

[6]. Sergueeva AV, Song C, Valiev RZ, Mukherjee K, Structure and properties of amorphous and nanocrystalline NiTi prepared by severe plastic deformation and annealing, Mater. Sci. Eng. A 339 (2003) 159-165.

[7].Tsuchiya K, Inuzuka M, Tomus D, Hosokawa A, Nakayama H, Morii K, Todaka Y, Umemoto M, Martensitic transformation in nanostructured TiNi shape memory alloy formed via severe plastic deformation, Mater. Sci. Eng. A. 438 (2006) 643-648.

[8]. Pushin VG, Stolyarov VV, Valiev RZ, Lowe TC, Zhu YT, Nanostructured TiNi-based shape memory alloys processed by severe plastic deformation, Mater. Sci. Eng. A. 410 (2005) 386-389.

[9]. Waitz T, Tsuchiya K, Antretter T, Fischer FD, Phase transformations of nanocrystalline martensitic materials, MRS Bull. 34 (2009) 814-821.

[10]. San Juan J, Nó ML, Superelasticity and shape memory at nano-scale: Size effects on the martensitic transformation, J. Alloys Compd. 577 (2013) S25-S29.

[11]. Singh R, Divinski SV, Rosner H, Prokofiev EA, Valiev RZ, Wilde G, Microstructure evolution in nanocrystalline NiTi alloy produced by HPT, J. Alloys Compd. 509 (2011) S290-S293.

[12]. Malard B, Pilch J, Sittner P, Delville R, Curfs C, In situ investigation of the fast microstructure evolution during electropulse treatment of cold drawn NiTi wires, Acta Materialia. 59 (2011) 15421556. 
[13]. Peterlechner M, Bokeloh J, Wilde G, Waitz T, Study of relaxation and crystallization kinetics of NiTi made amorphous by repeated cold rolling, Acta Materialia. 58 (2010) 6637-6648.

[14]. Demers V, Brailovski V, Prokoshkin SD, Inaekyan KE, Optimization of the cold rolling processing for continuous manufacturing of nanostructured Ti-Ni shape memory alloys, Journal of Materials Processing Technology. 209 (2009) 3096-3105.

[15]. Delville R, Malard B, Pilch J, Sittner P, Schryvers D, Microstructure changes during nonconventional heat treatment of thin $\mathrm{Ni}-\mathrm{Ti}$ wires by pulsed electric current studied by transmission electron microscopy, Acta Materialia. 58 (2010) 4503-4515.

[16]. Peterlechner M, Waitz T, Karnthaler HP, Nanocrystallization of NiTi shape memory alloys made amorphous by high-pressure torsion, Scripta Mater. 59 (2008) 566-569.

[17]. Huang X, Ramirez AG, Structural relaxation and crystallization of NiTi thin film metallic glasses, Appl. Phys. Lett. 95 (2009) 121911.

[18]. Klug HP, Alexander LE. X-ray Diffraction Procedures for Polycrystalline Materials, Wiley, New York, 1974.

[19]. Williamson GK, Hall WH, X-ray line broadening from filed aluminium and wolfram, Acta Metall. 1 (1953) 22-31.

[20]. Yavari AR, Moulec AL, Inoue A, Nishiyama N, Lupu N, Matsubara E, Botta WJ, Vaughan G, Di Michiel M, and Kvick A, Excess free volume in metallic glasses measured by X-ray diffraction, Acta Mater. 53 (2005) 1611-1619.

[21]. Ota K, Botta WJ, Vaughan G, Yavari AR, Glass transition $T_{g}$, thermal expansion, and quenchedin free volume $\Delta \mathrm{V}_{\mathrm{f}}$ in pyrex glass measured by time-resolved $\mathrm{X}$-ray diffraction, J. Alloys Compd. 388 (2005) L1-L3.

[22]. McGreevy RL, Pusztai L, Reverse Monte Carlo simulation: a new technique for the determination of disordered structures, Molecular Simulation, 1 (1988) 359-367.

[23] Bachir Aoun, A Rigid Body Reverse Monte Carlo Modelling Package Enabled with Machine Learning and Artificial Intelligence. J. Comput. Chem. (accepted)

[24] Bachir Aoun, The code of fullrmc, https://github.com/bachiraoun/fullrmc.

[25]. Rietveld HM, Line profiles of neutron powder-diffraction peaks for structure refinement, Acta Cryst. 22 (1967) 151-151.

[26]. Lutterotti L, Matthies S, Wenk H-R, Schultz AS, Richardson Jr JW, Combined texture and structure analysis of deformed limestone from time-of-flight neutron diffraction spectra, J. Appl. Phys. 81 (1997) 594-600.

[27]. Wenk H-R, Lutterotti L, Vogel SC, Texture analysis with the new HIPPO TOF diffractometer, Nucl. Instr. Meth. A. 515 (2003) 575-588.

[28]. Bernal JD, Geometry of the structure of monatomic liquids, Nature. 185 (1960) 68-70.

[29]. Bernal JD, The structure of liquids, Proc. R. Soc. A. 280 (1964) 299-322. 
[30]. Schwarz RB, Petrich RR, Saw CK, The synthesis of amorphous NiTi alloy powders by mechanical alloying, J. Non-crystalline Solids. 76 (1985) 281-302.

[31]. Yamauchi K, Nishida M, Itai I, Kitamura K, Chiba A, Specimen Preparation for Transmission Electron Microscopy of Twins in B19' Martensite of Ti-Ni Shape Memory Alloys, Mater. Trans., JIM 37 (1996) 210-217.

[32]. Willemse PF, Koopman BJ, Beyer J. The texture development of TiNiCu wire during thermomechanical cycling, J. de Physique 1 (1991) 329-333.

[33]. Gall K, Tyber J, Brice V, Frick CP, Maier HJ, Morgan N. J. Biomed, Tensile deformation of NiTi wires, Mater. Res. Part A. 75 (2005) 810-823. 


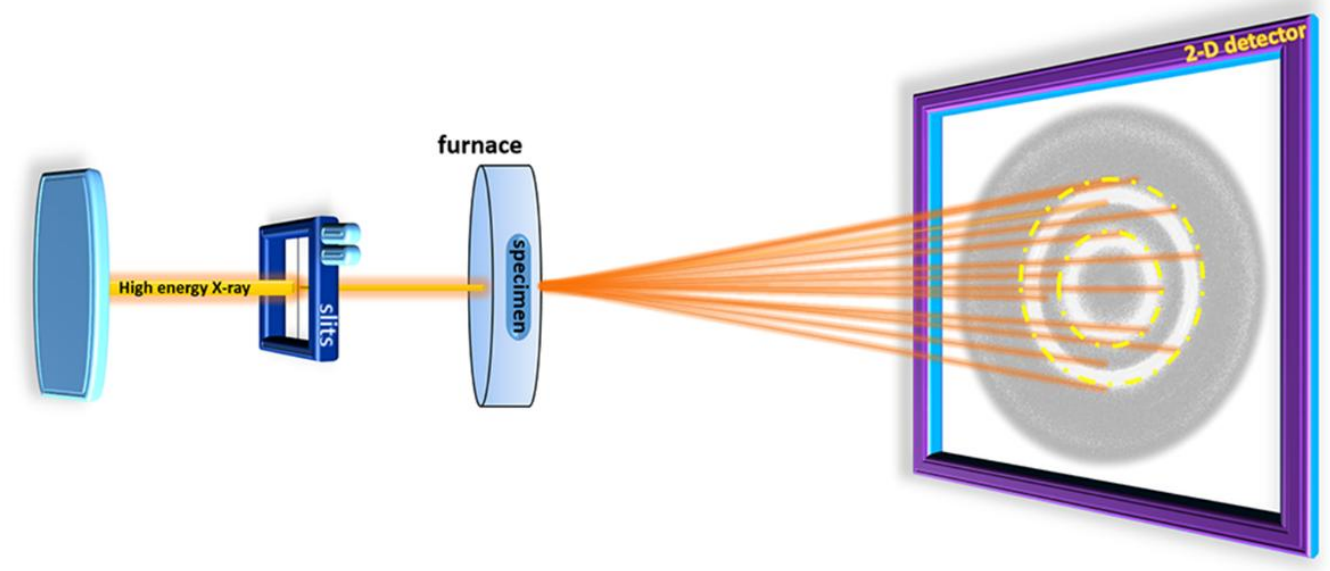

Fig. 1. Schematic illustration of in-situ high energy X-ray diffraction measurements. 

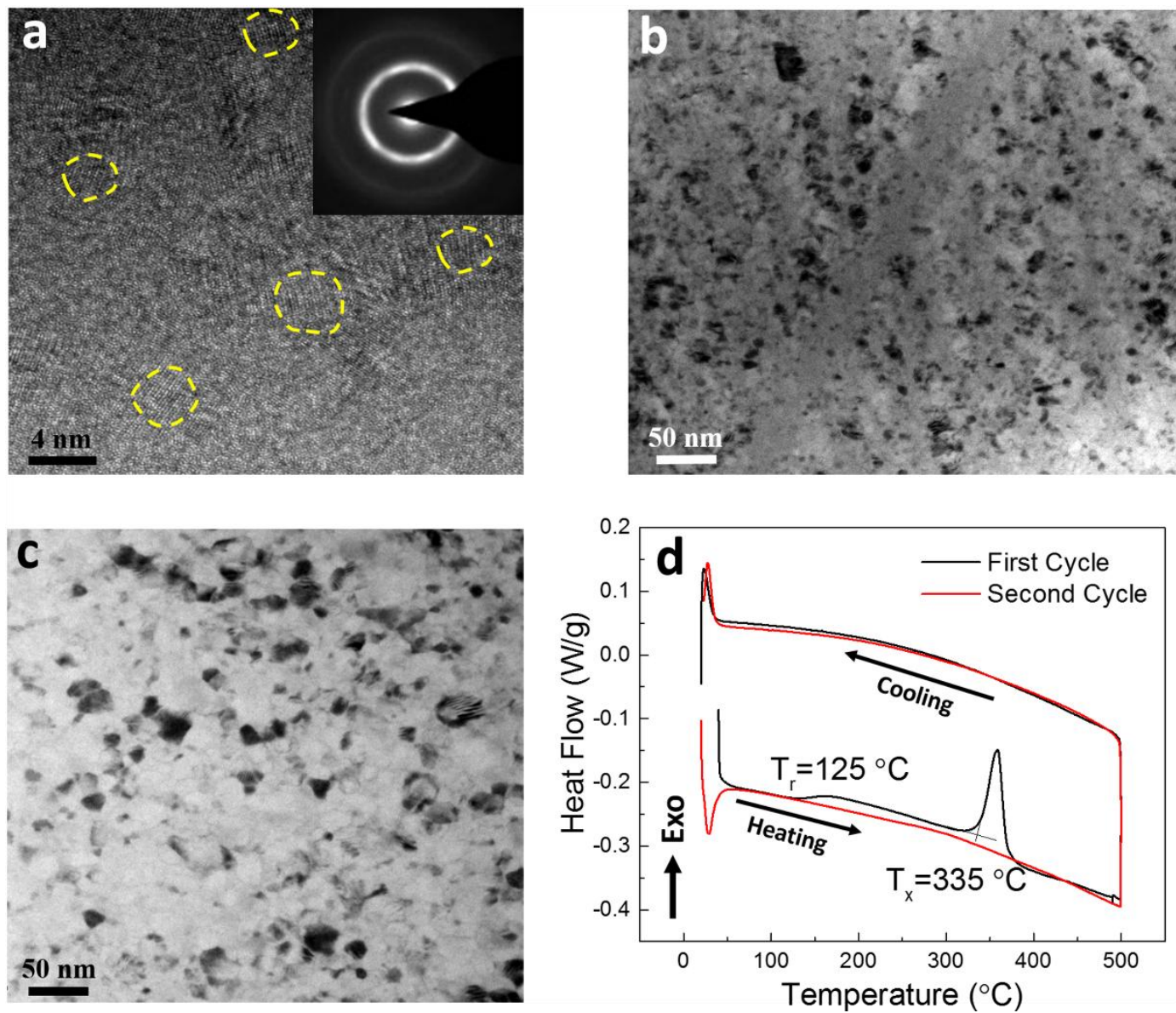

Fig. 2. TEM micrographs of the NiTi wire: (a) after cold drawing, (b) after heating to $350{ }^{\circ} \mathrm{C}$ for $2 \mathrm{~min}$, and (c) after heating to $450{ }^{\circ} \mathrm{C}$ for $2 \mathrm{~min}$, (d) differential scanning calorimetric curve of the cold drawn NiTi wire upon heating to $500{ }^{\circ} \mathrm{C}$. 

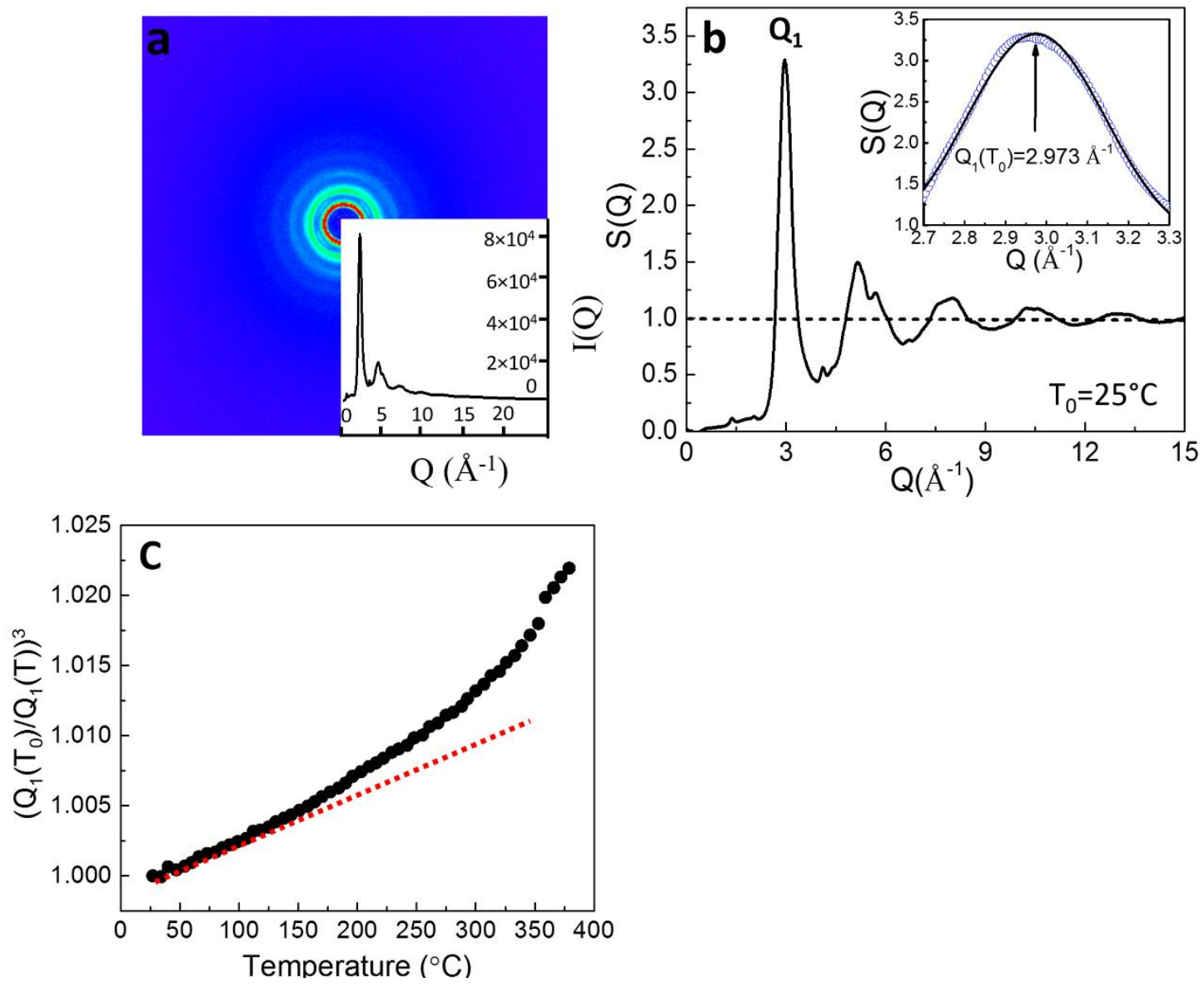

Fig. 3. Total structure factor and atomic volume change upon heating. (a) A high angle X-ray scattering pattern and integrated intensity curve of a cold drawn TiNi wire at $\mathrm{T}_{0}=25^{\circ} \mathrm{C}$. (b) Total structure factor $\mathrm{S}(\mathrm{Q})$ calculated from $\mathrm{I}(\mathrm{Q})$ as shown in (a) using Eq. 2. The first structure factor maximum is located at a scattering vector of $Q_{1}\left(T_{0}\right)=$ $2.973 \AA^{-1}$. The inset shows the Gaussian function fit (solid line) used to determine the peak parameters. (c) Relative atomic volume changes $\left[Q_{1}\left(T_{0}\right) / Q_{1}(T)\right]^{3}=V / V_{0}$ during heating from $25^{\circ} \mathrm{C}$ to $380^{\circ} \mathrm{C}$. 

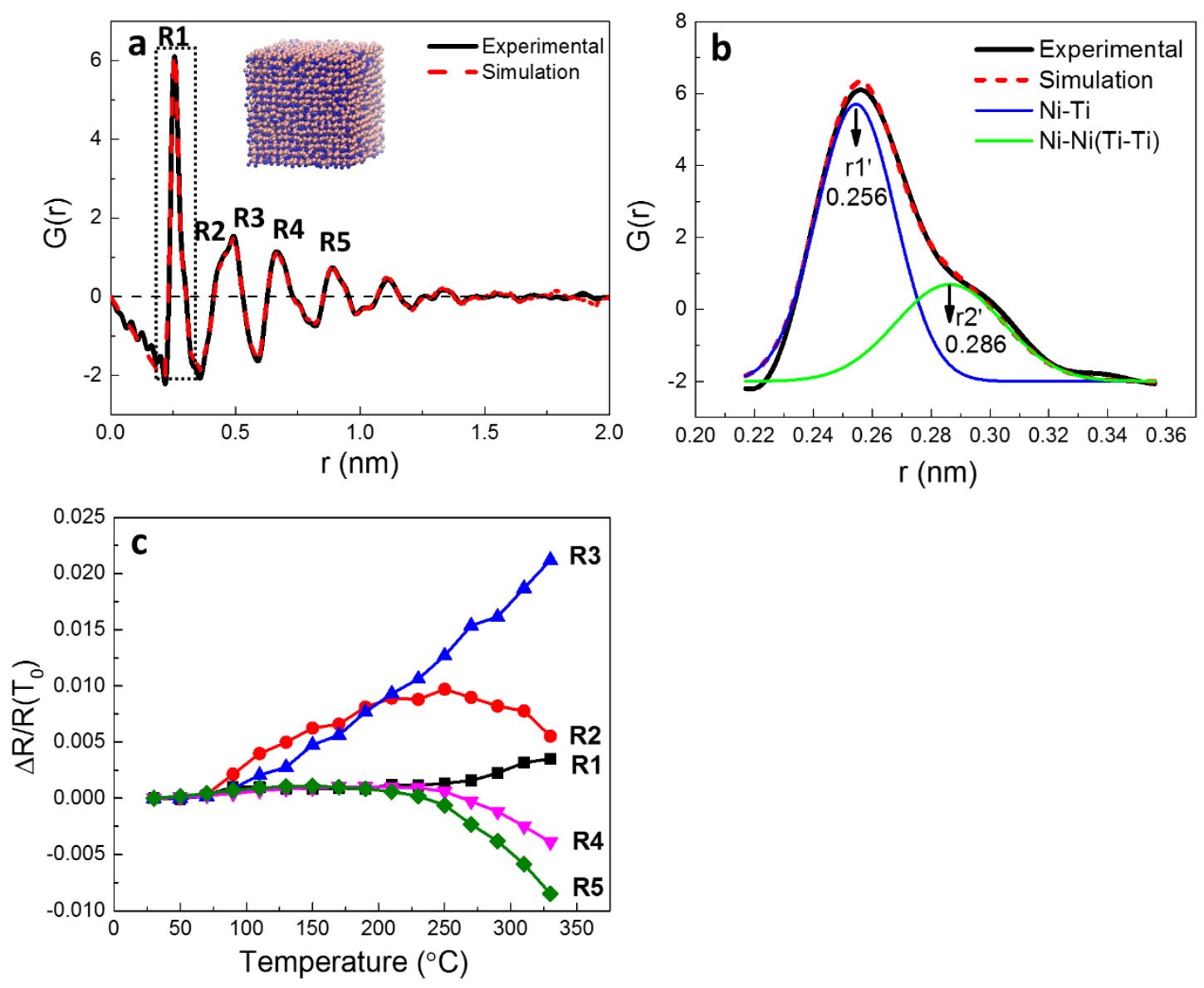

Fig. 4. Atomic rearrangement during structural relaxation. (a) Reduced pair distribution function $\mathrm{G}(\mathrm{r})$ of the cold drawn TiNi wire determined from the measured $\mathrm{S}(\mathrm{Q})$ at $\mathrm{T}_{0}=25^{\circ} \mathrm{C}$ and a reverse Monte Carlo simulation. (b) An enlarged view of the R1 peak and its deconvolution. (c) Relative changes of bond lengths during heating. 

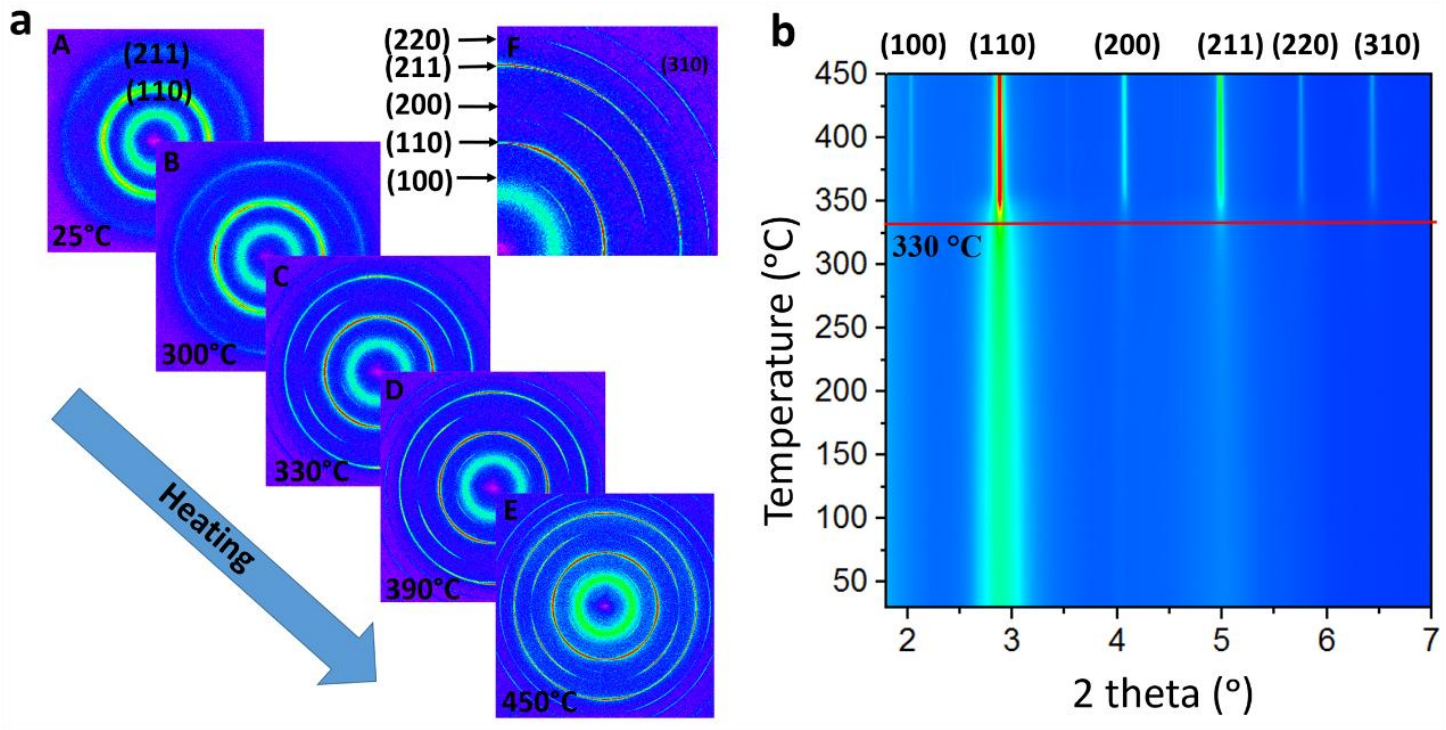

Fig. 5. High energy XRD analysis of structural evolution of cold draw NiTi during heating. (a) A selection of 2D diffraction patterns at different temperatures; (b) Evolution of diffraction peaks of the cold drawn NiTi wire during heating. 


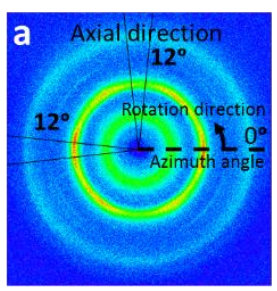

$25^{\circ} \mathrm{C}$
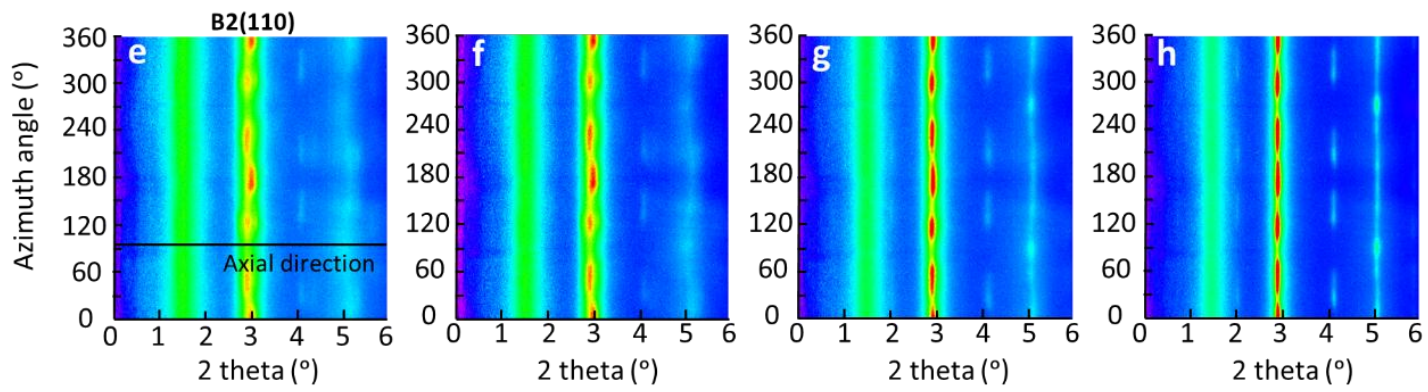

Fig. 6. Comparison of 2D diffraction patterns and the corresponding azimuthally $\left(0-360^{\circ}\right)$ unrolled diffraction images of the NiTi wire under different temperatures. 

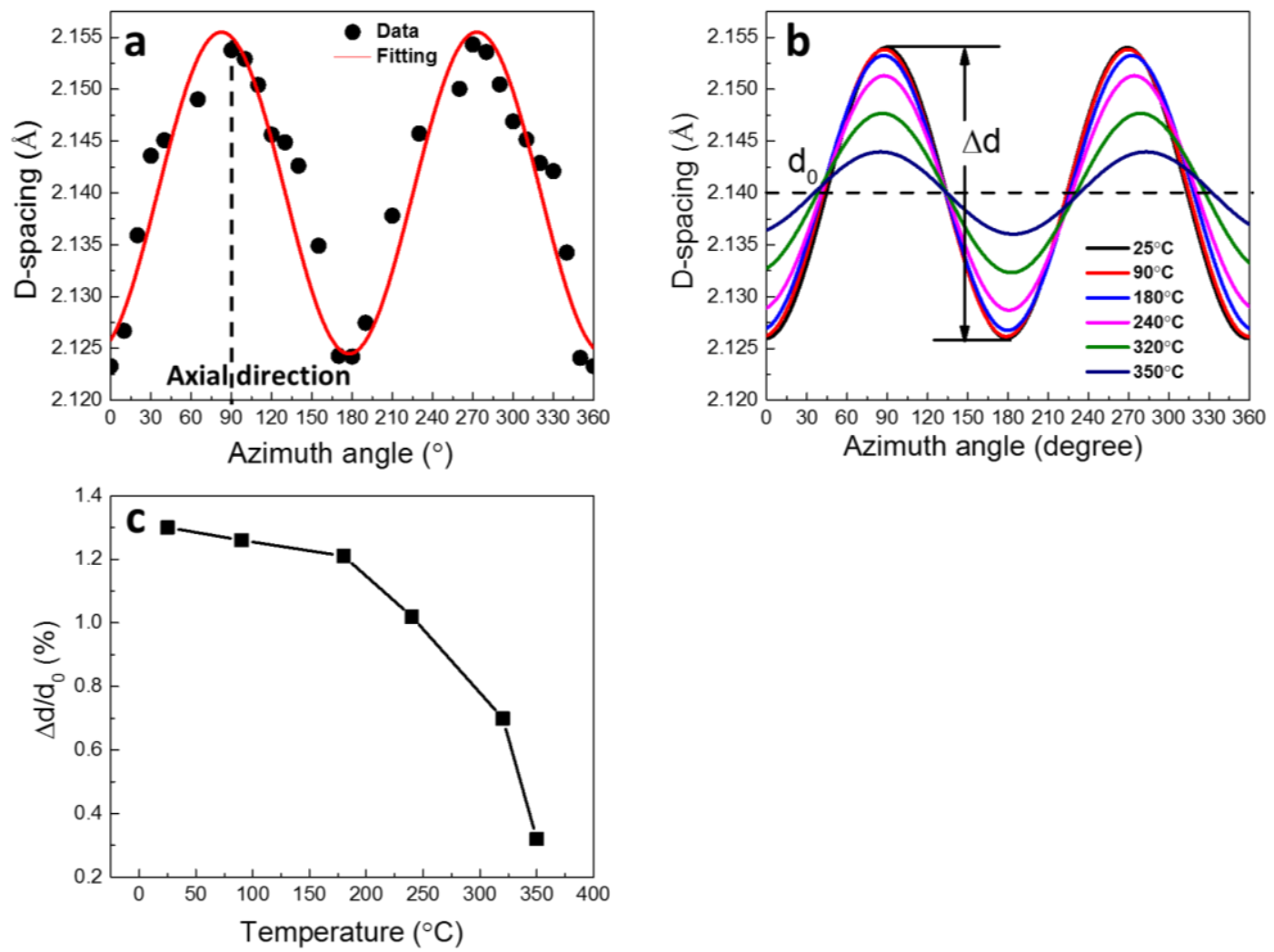

Fig. 7. Relaxation of B2(110) lattice strain anisotropy in the nano-crystalline domains in the cold drawn NiTi wire during heating. (a) B2(110) d-spacing verses azimuth angle. (b) Comparison of anisotropic d-spacing in samples heated to different temperatures. (c) Evolution of B2(110) lattice strain anisotropy during heating. 

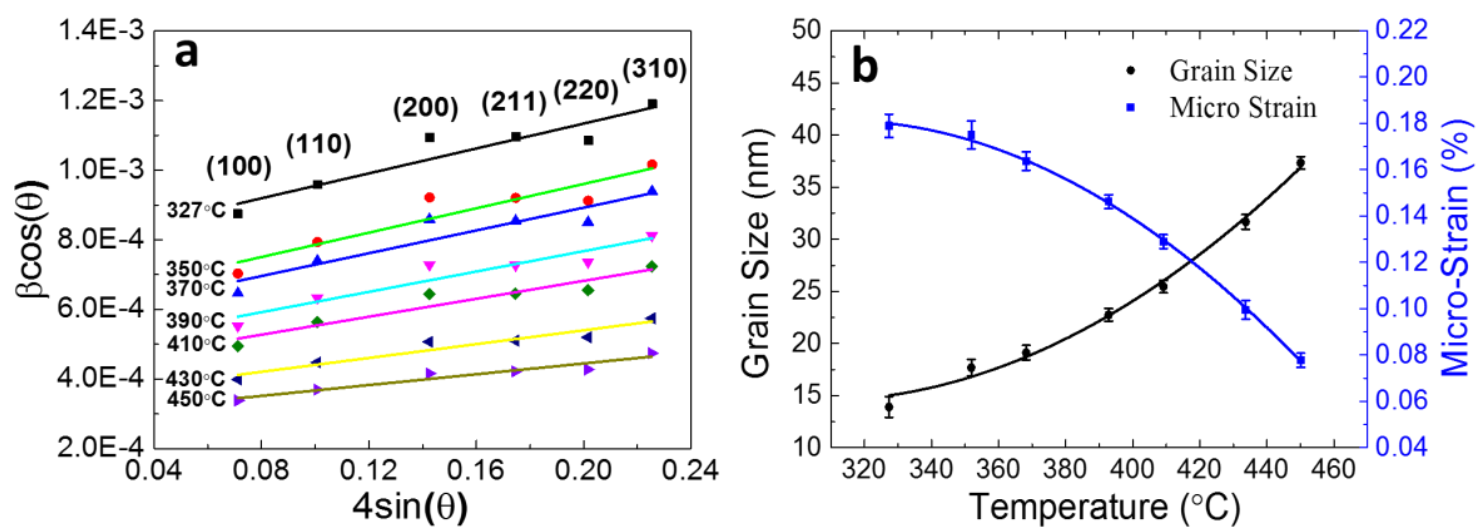

Fig. 8. Williamson-Hall Plot analysis. (a) Williamson-Hall Plot of the cold drawn NiTi wire at different temperatures. (b) Evolution of grain size and micro strain after crystallization. 


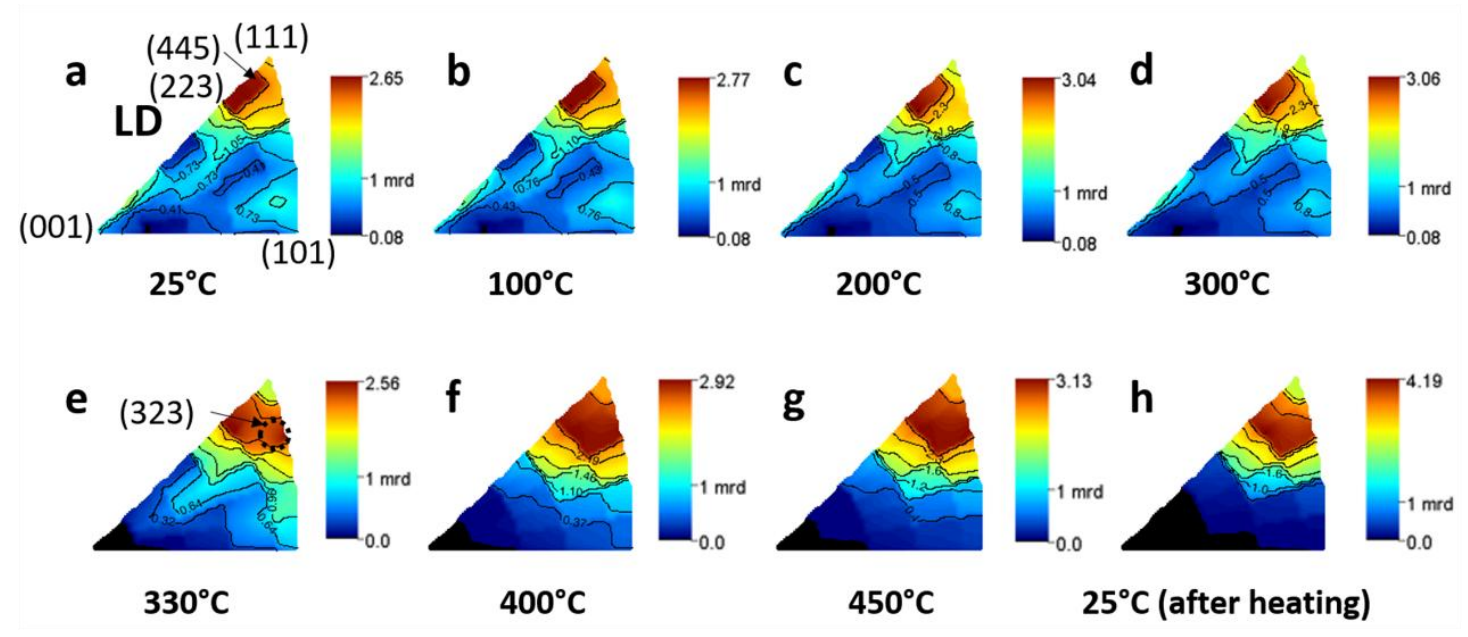

Fig. 9. Comparison of inverse pole figures along the longitudinal direction at different heating temperatures. 
Table 1. Atomic arrangement of ideal HCP and BCC structure in short range of $\mathrm{r}(\mathrm{r}<$ $0.661 \mathrm{~nm}$ ) and atomic rearrangement from $\mathrm{BCC}$ to $\mathrm{HCP}$ during amorphization (marked by red and blue arrows).

\begin{tabular}{|c|c|c|c|c|c|c|c|c|c|c|c|c|c|c|}
\hline & $r(n m)$ & $\begin{array}{c}\mathrm{r} 1 \\
0.256\end{array}$ & $\begin{array}{c}r 2 \\
0.296\end{array}$ & $\begin{array}{c}r 3 \\
0.362\end{array}$ & $\begin{array}{c}r 4 \\
0.418\end{array}$ & $\begin{array}{c}\mathrm{r} 5 \\
0.443\end{array}$ & $\begin{array}{c}r 6 \\
0.490\end{array}$ & $\begin{array}{c}r 7 \\
0.512\end{array}$ & $\begin{array}{c}\mathrm{r} 8 \\
0.572\end{array}$ & $\begin{array}{c}r 9 \\
0.591\end{array}$ & $\begin{array}{c}\mathrm{r} 10 \\
0.609\end{array}$ & $\begin{array}{c}r 11 \\
0.627\end{array}$ & $\begin{array}{c}\mathrm{r} 12 \\
0.644\end{array}$ & $\begin{array}{c}\text { r13 } \\
0.661\end{array}$ \\
\hline \multirow{3}{*}{ ํㅗㄴ } & No. of Atoms & 12 & & 6 & 2 & 18 & 12 & 6 & 12 & & 12 & 6 & 6 & 12 \\
\hline & Total No. Atoms & 12 & 12 & 18 & 20 & 38 & 50 & 56 & 68 & 68 & 80 & 86 & 92 & 104 \\
\hline & Direction Indices & $\begin{array}{l}1 / 21 / 2 \overline{10} \\
01 / 21 / 23 / 4\end{array}$ & & $\overline{1} 103 / 4$ & $000^{3} / 2$ & $\begin{array}{l}3 / 2 \frac{3}{3} 2^{000} \\
1 / 22^{3 / 2} 2^{3} / 4\end{array}$ & ${ }_{1 / 2}^{1 / 2} 2^{3} / 2$ & $11 \overline{2} 0$ & $3 / 2^{2} 2^{1 / 2} 2^{3 / 4}$ & & $3 / 2^{3} / 2^{3} 0^{3 / 2}$ & $\overline{2} 023 / 4$ & $01 / 2 \overline{1}^{1 / 2} 9 / 4$ & $\overline{1} 2 \overline{1} 3 / 2$ \\
\hline & & & & & 46 & $\rightarrow$ & 12 & 2 & $\rightarrow 4$ & 6 & $12_{\leftarrow}$ & $6 \%$ & 5 & 48 \\
\hline \multirow{3}{*}{ 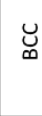 } & No. of Atoms & 8 & 6 & & 12 & & 24 & 8 & & 6 & & & 24 & 24 \\
\hline & Total No. Atoms & 8 & 14 & 14 & 26 & 26 & 50 & 58 & 58 & 64 & 64 & 64 & 88 & 112 \\
\hline & Direction Indices & $1 / 22^{1 / 2} 2^{1 / 2}$ & 100 & & 110 & & $3 / 21 / 21 / 2$ & 111 & & 200 & & & $3 / 21 / 23 / 2$ & 210 \\
\hline \multicolumn{2}{|c|}{ Measured r value $(\mathrm{nm})$} & $\begin{array}{c}\text { R1 } \\
0.256\end{array}$ & & & \multicolumn{2}{|c|}{$\begin{array}{c}\text { R2 } \\
0.427\end{array}$} & $\begin{array}{c}\text { R3 } \\
0.494\end{array}$ & & & & & & & \begin{tabular}{|c|} 
R4 \\
0.663
\end{tabular} \\
\hline
\end{tabular}



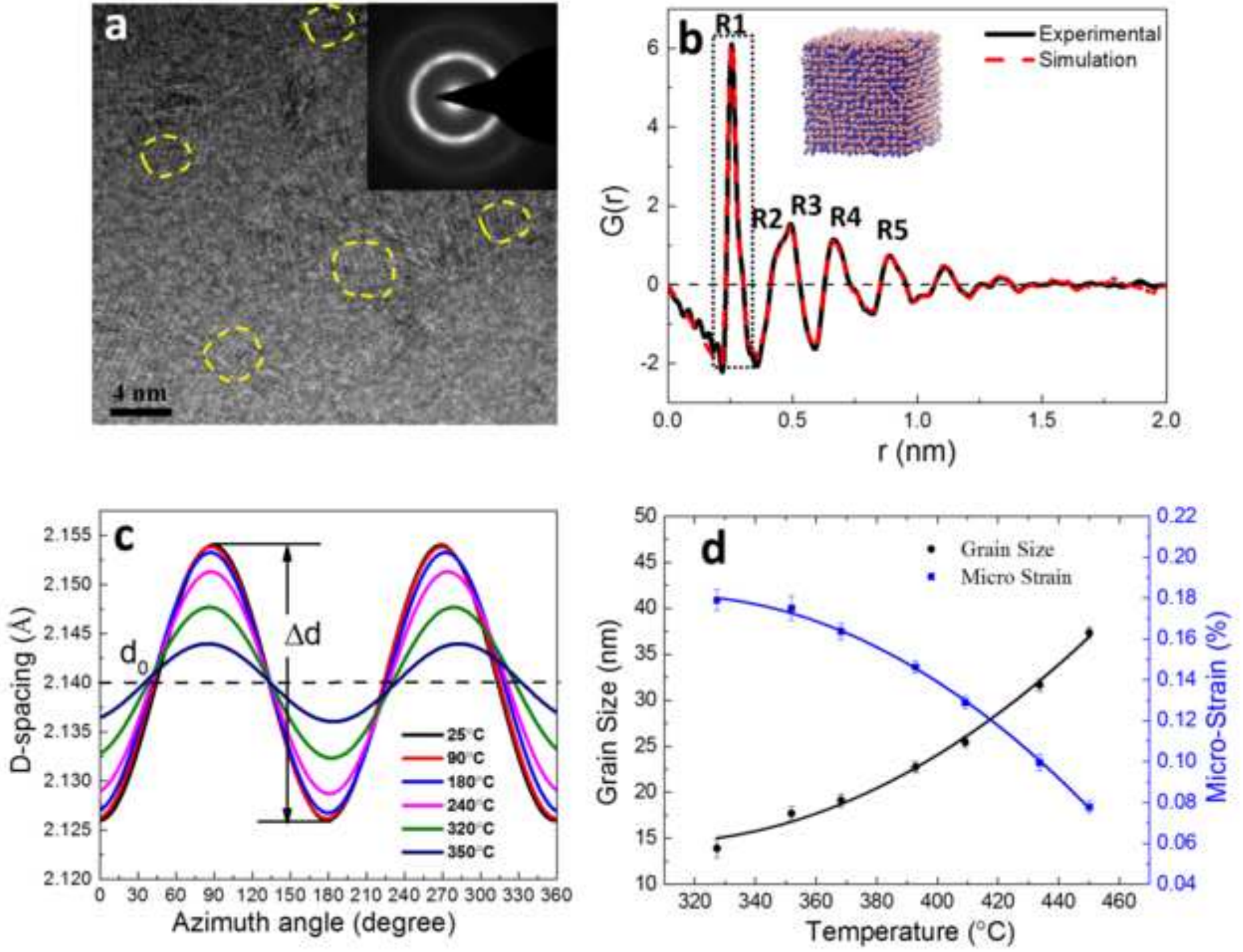